\title{
Damage Control Surgery: Management of Critically Injured Patients
}

\author{
Rawal, S.t
}

\section{Abstract}

It has become apparent that extensive and prolonged surgery performed on a critically injured patient, especially following trauma, the results are poor even in experienced hands. The traditional approach to resuscitation after abdominal trauma has been based on tried and tested methods of managing penetrating injury with exploration, control of bleeding and contamination and attempts to achieve definite repair of damaged structures. Exsanguination, haemorrhage following Severe trauma leads to the onset of a cycle of interrelated variables; metabolic acidosis, profound hypothermia, and clinically obvious coagulopathy. Each factor reinforces the other to form a 'bloody vicious cycle'leading to death as a result of irrevesible metabolic insult. These observations have lead to the development of a new surgical strategy that sacrifices the completeness of immediate repair in order to adequately address the combined physiological impact of trauma and surgery. This approach consists of three phases, initially to control exsanguination and prevent spillage of intestinal contents and urine; second phase of secondary resuscitation in ICU unit with ventillatory support and the third phase where the abdominal packings are removed with definite repair of abdominal injuries. The 'damage control' concept has been shown to increase overall survival and is likely to modify the management of critically injured patients.

\section{Introduction}

The introduction of complex surgeries to manage penetrating injuries were a salient feature of trauma surgery in the 1960s and 1970s. However, over the past decade it became apparent that the results of such extensive and prolonged surgery in a critically injured patient were not just unsatisfactory but lead to a high degree of morbidity and mortality even in

\footnotetext{
* Dr. Sushil Rawal. MBBS

Major, MS resident (NAMS)

Shree Birendra Hospital, Chhauni
}

experienced hands. The triad of hypothermia, metabolic acidosis and coagulopathy limits the patients ability to cope with the physiological consequences of trauma and this can cause a failure of even the most technically successful repair to save the life of the patient. These observations lead to the development of a new surgical strategy, phrase adopted by Schwab and his co-workers in Pennsylvania, to describe the evolving concept of saving life after major trauma by deferring treatment of anatomical disruptions and focusing on restoring the patients physiology.

Damage control is a term used by the US Navy that describes the capacity of a ship to absorb damage and maintain mission integrity. For the trauma surgeon damage control consists of carrying out appropriate maneuvers to achieve initial injury control.

The outcome of damage control approach depends on careful selection of patients and timing based on pathophysiology. Optimal results are achieved by early identification of patients who require damage control.

Most patients with penetrating abdominal injury require traditional management, with the damage control approach being necessary in only a few. In a busy urban trauma unit, only approximately $8 \%$ of patients undergoing laparotomy required damage control. The clinical manifestations of hemodynamic instability, hypotension, tachycardia, tachypnea, and altered mental status are indications for the potential need for damage control. Patients with major abdominal vascular injury and multiple visceral injury are candidates for damage control. Despite its simplicity and logic, this new approach is being adopted slowly, only during the last decade reports have started to emerge on the use of damage control.

\section{Historical Retrospective}

The concept of rapid termination of surgery after intra-abdominal packing in the face of massive hemorrhage followed by delayed re-exploration for 
definitive repair has been a part of the surgeons armamentarium. Alternate techniques included packing of exsanguinating injuries and rapid closure using towel clips. Pringle in 1908 focused on the management of hepatic hemorrhage using sutures above gauge packing, was later modified by Halsted in 1913.Packing for control of hepatic hemorrhage fell out of favour and, by the end of world war II, was almost universally condemned. In 1955 , Madding wrote that temporary packing may be effective for checking bleeding, but emphasized on the removal of packs prior to completion of surgery. By 1970s, a few centres were using this technique in selected situations to arrest hemorrhage. In 1983, Stone et al. Described a stepwise approach using intra-abdominal packing and rapid termination of laparotomy with temporizing maneuvers for other injured viscera. In 1993, Rotondo et al. Introduced the term "damage control" and detailed a standardized approach which yielded a $58 \%$ survival rate. Recently, the damage control concept has been shown to increase overall survival and is likely to modify the management of the critically injured patient.

\section{Damage Control For Abdominal Trauma}

Damage control is defined in three distinct phases. The first phase consists of the initial surgical approach where a full midline incision is made, hemoperitoneum is evacuated, abdomen is packed in four quadrants at the dissected surfaces and injured organ. Further control of hemorrhage is achieved by ligation, clamping, or balloon tamponade followed by rapid closure of the skin. The key principle is that packing should reapproximate disrupted tissue planes. Overpacking will result in raised intra-abdominal pressure and hemodynamic instability due to compression of inferior vena cava, producing abdominal compartment syndrome. Underpacking will fail to control bleeding.

Hollow viscus injuries are controlled by tying with tapes, simple running sutures or by the use of stapling devices, reconstruction is deferred.Biliary, pancreatic and urological injuries are controlled by external tube drainage and stenting.

Damage control phase II consists of secondary and continued resuscitation of the patient in ICU. Require maximization of hemodynamics, core rewarming, correction of coagulopathy, complete ventillatory support and further identification period of $36 \mathrm{hr}$ is ideal to allow for ad homeostasis. Unscheduled reoperation required if surgical control of bleeding has $m_{\text {iy }}$; achieved or if visceral edema has occurred consequence of ischemia reperfusion syndrome Danage control phaseIII consists of re-operation removal of packs, definite repair of abdom injuries, and closure when the patient has beenf: resuscitated, is warm, and the coagulopathy been restored, usually $24-48 \mathrm{hrs}$ after after the in intervention. At times it may be necessary to to $p$ a second time and proceed to temporary closuri:

\section{Patient Selection For Damage Contri Surgery}

Damage control surgery is indicated for onh limited number of critically wounded patients, sthe major problem is deciding when it: appropriate.Correct selection is critical, as ear identification of those patients who require dam: control promotes optimal results.Patients wi complex injuries such as major abdominovascu'? injuries.

Multiple visceral injuries, multifocal an multicavitary sources of exsanguination,represer: suitable candidates. Intraoperative, injury patte: recognition is most important when making $b$ decision for damage control approach. Other critre factors for damage control include a $\mathrm{pH}$ of $<7,3$. core temperature of $<35 \mathrm{C}$ and coagulopath: Operative time of $>90 \mathrm{~min}$ and a transfusin requirement of at least 10 units of packed cellsts also indicative factors.

\section{Conclusions}

Patients with gravely traumatic wounds at admitted to trauma centers, and the operating lest has very limited time to stabilize the patients. has become apparent that prolonged and extensit surgical procedures on the critically injured is ofls poor even in experienced hands Damage conth surgery sacrifices the completeness of th immediate repair so as to adequately address combined physiological impact $f$ trauma gri surgery. The decision to abbreviate laparotont? should ideally made within the first few minuter the surgery. The success of this strategy depent on the correct selection of patients and the decis sil 
as fe explore the abdomen. Team coordination and time matugement are becoming the key elements for promoting patient survival.

\section{References:}

1. Stone HH, Fabian TC, SatianiB.Experiences in the management of pancreatic trauma.J Trauma 1981:21:257-61,

2. Rotondo MF, Schwab CW. Damage control: an appronch for improved survival in exsanguinating penetrating abdominal injury.J Trauma 1993;35:375-83.
3. Trunkey DD. History and development of Irauma care in United States. ClinOrthop2000;374;36-46.

4. Hirshberg A,Mattox KL. Planned reoperation for severe trauma.Ann Surg 1995;222:3-8

5. Madding G. Injuries of the liver. Arch Surg 1955;70:748-53. 